\title{
Beobachtungen über Vorkommen, Abundanzen und Körpergrößen von mediterranen Arten der Gattung Astropecten
}

\author{
G. Ribi, H. Burla \& P. Ochsner \\ Zoologisches Musetum der Universität Zürich; \\ Zürich, Schweiz
}

\begin{abstract}
Observations on occurrence, abundance and body sizes of Mediterranean species of the genus Astropecten (Asteroidea, Echinodermata). At various coastal positions of the western Mediterranean starfishes of the genus Astropecten were recorded. The largest of the six species, $A$, aranciacus, proved to be a generalist in occurrence; however, its abundance is exceeded by other Astropecten species at various positions. It appears that the sand quality influences the local abundance of $A$. aranciacus. Water depth is also influential. This is shown not only by the zonation of the species studied, but also by special frequency distributions of body sizes as related to water depth. The shaliow water areas near the beach may be a refuge for juveniles of $A$. bispinosus. The frequency distribution of body sizes for A. jonstoni is usually bimodal. For the smaller mode, samples taken at different times yielded a growth rate of $4 \mathrm{~mm}$ in 37 days.
\end{abstract}

\section{EINLEITUNG}

Von den sechs Seestern-Arten der Gattung Astropecten, die im Mittelmeer nachgewiesen sind (Tortonese, 1965), kommen fünf, nämlich $A$ aranciacus, A. bispinosus, A. jonstoni, $A$. irregularis und $A$. platyacantbus an mancher Küstenstelle auf Sandboden vor. Okologisch sind Seesterne von erheblicher Bedeutung. Als Räuber, die sich von anderen Makro Invertebraten des Benthals ernähren, besetzen sie in der Nahrungskette obere Ränge und regulieren wohl die Bestandesdichte ihrer Beutetiere (Massé, 1966; Paine, 1969; Brun, 1968; Gulliksen \& Skieveland, 1973; ChristenSEN, 1970; LAXTON, 1974; DANA et al., 1972). In ihrer eigenen Bestandesdichte kommen die allgemeinen trophischen Bedingungen, die in dem von ihnen besetzten Areal herrschen, zum Ausdruck. In der vorliegenden Arbeit wird über die Abundanzen der drei Seestern-Arten $A$. aranciacus, A. bispinosus und $A$. jonstoni an verschiedenen Küstenstellen berichtet. Je nach der Methode, die angewandt wurde, ergaben sich Schätzungen der absoluten oder relativen Bestandesdichte. Da nicht auszuschließen ist, daß sich Seesterne verschiedener Größenklassen ökologisch uneinheitlich verhalten, wurde ihre Körpergröße ebenfalls protokolliert. Erfahrungen über die Häufigkeitsverteilung der Körpergröße bilden den zweiten Teil der Ergebnisse. 
Tabelle 1 informiert über die Gebiete, in denen Meß- und Zähldaten gewonnen wurden sowie über das Vorkommen der Arten. Andere Küstenstellen, an denen das Sammeln unergiebig war, sind hier ausgelassen. Abbildung 1 zeigt die geographische Lage der Arbeitsgebiete. In Tabelle 1 bezeichnen die Tiefenangaben die Zone, in denen Astropecten-Arten gefunden wurden. In der Regel wurde die untere Zonengrenze will-

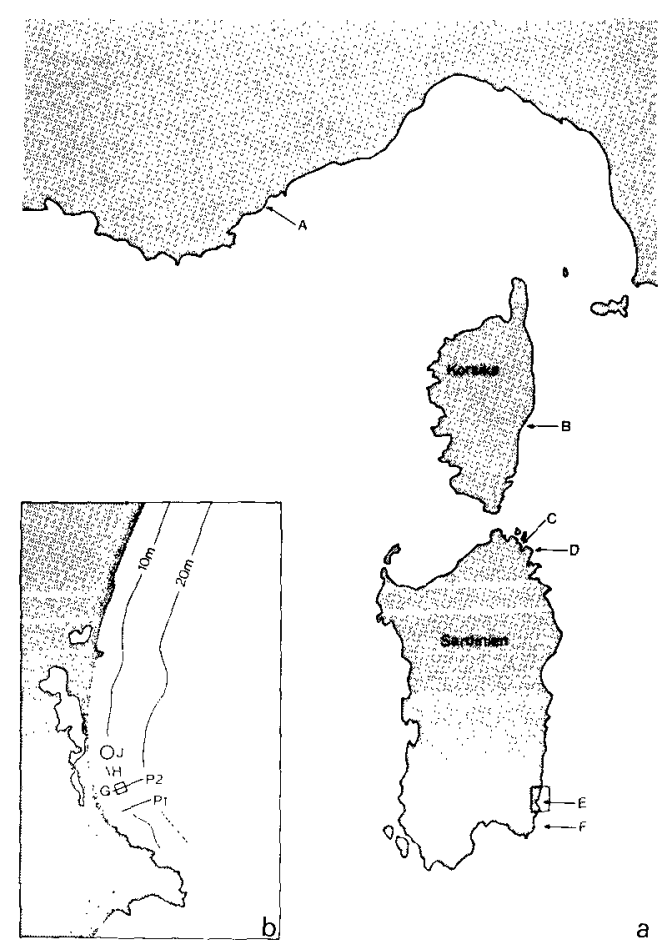

Abb. 1a: Ubersicht über die Untersuchungsgebiete: $A=$ Le Lavandou, $B=$ Ghisonaccia, $C=$ Cannigione, $\mathrm{D}=$ Golfo Pevero, $\mathrm{E}:=$ Costa Colostrai, $\mathrm{F}=$ Costa Rei

Abb. 1b: Anordnung der Profile und Sammelstellen an der Costa Colostrai. P 1 und P 2 sind Profilc, entlang derer Seesterne gesammelt wurden; sie reichen von 3 bis $20 \mathrm{~m}$ Tiefe. G ist ein Quadrat von $80 \mathrm{~m}$ Seitenlänge, auf dem die absolute Seesterndichte durch $\Lambda$ bsammeln bestimmt wurde. Bei H wurde die Seesterndichte mit der Egge ermittelt. Bei J wurde Astropecten bispinosus gesammelt

kürlich da gesetzt, wo die Abundanzen sich stark verringerten. In allen Gebieten wurde Sandgrund abgesucht. Im übrigen waren die Gebiete in mancher Beziehung uneinheitlich. Bei Cannigione wachsen Posidonia-Rasen unterhalb einer Tiefe von 6 Metern, wobei im oberen Teil einer breiten Grenzzone die Posidonien nur inselartig vorkommen, im unteren Teil dagegen überwiegen und inselartige Sandflächen einschließen. An der Costa Colostrai stehen stellenweise lockere Zostera-Bestände über den Sandboden verstreut, Posidonien fehlen, so daß der Grund von der Strandlinie bis in eine Tiefe von 20 Metern überblickt werden kann. 


\section{METHODEN}

Die Tiere wurden mittels Gerätetauchen gesucht. Die Arten der Gattung Astropecten graben sich periodisch in den Sand ein, so daß nie alle Seesterne, die in einem Areal leben, gleichzeitig sichtbar sind. Um dennoch möglichst alle Tiere zu erfassen, wurde zu verschiedenen Tag- und Nachtzeiten getaucht.

Bei der Bestimmung der relativen Dichte wurde die Tauchzeit als Bezugsgröße gewählt. Wurde versucht, in einem Areal von bekannter Fläche alle Seesterne zu erfassen, ließ sich die absolute Dichte berechnen; sie wird angegeben als durchschnittliche Fläche pro Seestern. Absolute Dichtebestimmungen wurden erstens durch systematisches Absammeln der Seesterne auf einer begrenzten Fläche durchgeführt, zweitens mittels einer Egge, die vom Motorboot über den Grund gezogen wurde und den Sandboden etwa $5 \mathrm{~cm}$ tief durchkämmte. Ein Taucher folgte ihr und sammeite die aufgewühlten Seesterne ein. Mit diesem Gerät wurde im Sommer 1975 an der Costa Colostrai gearbeitet. Vermutlich ist es die verläßlichste Methode, hat aber den Nachteil, daß die Dornen der Egge größere Seesterne verletzen oder zerschneiden. Bei der anderen $\mathrm{Me}-$ thode, die absolute Dichte zu bestimmen, ist stets mit Seesternen zu rechnen, die während der ganzen Beobachtungsperiode nie zur gleichen Zeit aktiv waren wie die Taucher.

Im August 1975 wurde an der Costa Colostrai die absolute Dichte von A. bispinosus nach beiden Methoden bestimmt; sie ergaben übereinstimmende Werte.

Außer der Art und Größe der Seesterne wurden regelmäßig auch Datum, Tageszeit, Taucher, Gebiet, Küstenstelle, Tiefe und Vegetationsverhältnisse protokolliert. Bei zwei Projekten (Zeilen 4 und 5 in Tab. 1) wurden außerdem drei Klassen von Sandkorngrößen subjektiv unterschieden: feiner Sand, grober Sand und Kies. Für jede geschätzte Klasse wurde die Sandkorngröße stichprobenweise bestimmt. Für feinen Sand ergab sich eine durchschnittliche Korngröße von weniger als $1 \mathrm{~mm}$, für groben Sand von 1-3 $\mathrm{mm}$ und für Kies von mehr als $3 \mathrm{~mm}$.

\section{ERGEBNISSE}

\section{Vorkommen und Abundanzen}

Soweit aus der Literatur bekannt, überdecken sich im Untersuchungsgebiet die Verbreitungsareale der Astropecten-Arten, doch ist nicht zu erwarten, daß die Arten innerhalb dieses Gebietes an jeder Küstenstelle vorkommen. Im Vergleich der sechs Orte erweisen sich Le Lavandou und Cannigione mit fünf nachgewiesenen Arten als faunistisch reichhaltigste Gebiete, der Strand bei Ghisonaccia mit zwei Arten als faunistisch ärmstes. An der Ostküste Sardiniens wurden an fünf Sammelstellen drei oder vier Arten angetroffen. Das Ergebnis besagt, daß in manchem Gebiet, das für Astropecten günstig ist, zwei oder mehr Arten koexistieren.

In Sardinien lassen sich die faunistischen Unterschiede auf die Bodenverhältnisse zurückführen: auf Kies lebt fast nur $A$. aranciacus, auf Sand überwiegen $A$. bispinosus oder A. jonstoni. Bei einem früheren Projekt wurde der Einfluß des Sediments auf 


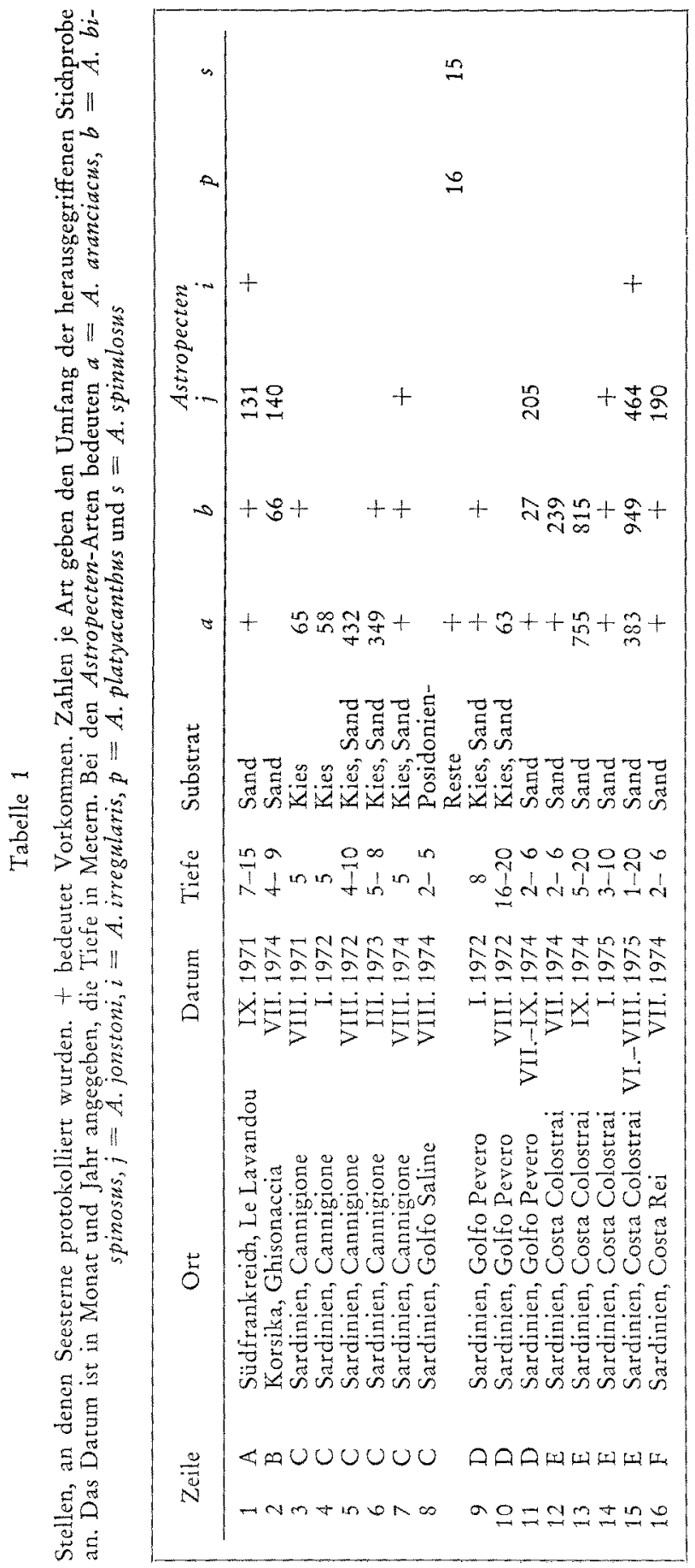


A. aranciacus untersucht. Auf einer Sandfläche von $1200 \mathrm{~m}^{2} \mathrm{Größe}$, angelegt im Januar $1972 \mathrm{im}$ Golfo Pevero (Tab. 1, Zeile 9), waren $500 \mathrm{~m}^{2}$ von feinem Sand bedeckt, der übrige Teil von Kies. Die meisten Seesterne der Art A. aranciacus fanden sich in der Grenzzone beider Flächenteile, wo Kies mit Sand vermischt war. Auf feinem Sand sowie auf Kies betrugen die absoluten Dichten ungefähr ein Seestern pro $100 \mathrm{~m}^{2}$, an der Grenzzone ein Seestern pro $25 \mathrm{~m}^{2}$.

Im Vergleich der Arten tritt $A$. aranciacus mit Regelmäßigkeit auf. Okologisch ist er als Generalist zu bezeichnen, der fast an jeder Küstenstelle, die sich nach Sediment und Wasserverhältnissen eignet, seine Nahrung findet (MAssé, 1966). Beachtet man die Abundanzverhältnisse, erwies sich $A$. jonstoni an vier Orten als dominierend, $A$. aranciacus an zwei Orten, $A$. bispinosus an nur einem Ort. An der Costa Colostrai hieiten sich $A$. aranciacus und $A$. bispinosus etwa die Waage, während $z u$ anderen Zeiten sowie an anderen Stellen und Orten die abundanteste Art zahlenmäßig die anderen stark übertriff. Offenbar gibt es unter Astropecten-Arten kein einheitliches Abundanzgefälle, und wir sind weit entfernt von einem Verständnis der Faktoren, die der einen oder anderen Art zum Dominieren verhelfen.

\section{Bestandsdichte}

Unter Vorbehalt darf die Anzahl der pro Minute gesichteten Seesterne als relative Dichte gelten. Das Maß exlaubt den Vergleich von Abundanzen, sei es zwischen Tauchstellen oder Arten. Im August 1972 wurde bei Cannigione auf sieben verstreut liegenden Tauchstellen die relative Dichte von $A$. aranciacus protokolliert. Im Durchschnitt wurde etwa alle drei Minuten ein Seestern registriert (Tab. 2). In den Dichtewerten

Tabelle 2

Relative Dichte von Astropecten aranciacus als durchschnittliche Anzahl gefundener Seesterne pro Tauchminute. Die Stellen 1 bis 5 liegen bei Cannigione (Zeile 5 in Tab. 1), die Stellen 6 und $7 \mathrm{im}$ Golfo Pevero (Zeile 10 in Tab. 1). Mit $\mathrm{n}$ ist die Anzahl Seesterne bezeichnet

\begin{tabular}{cccc|}
\hline Stelle & Tiefe $(\mathrm{m})$ & Seesterne $/ \mathrm{min}$ & $\mathrm{n}$ \\
\hline 1 & $4-6$ & 0,20 & 63 \\
2 & $4-6$ & 0,46 & 62 \\
3 & $5-10$ & 0,50 & 64 \\
4 & $6-7$ & 0,42 & 91 \\
5 & 8 & 0,57 & 133 \\
6 & 8 & 0,21 & 16 \\
7 & $16-20$ & 0,09 & 66 \\
\hline
\end{tabular}

erkennen wir den Einfluß von Tauchstelle und Tiefe. Mit der gleichen Methode wurden an der Costa Colostrai drei Arten verglichen (Tab. 3); diesmal auf der in Abbildung 1b gezeigten Anlage. Die Dichte variierte zwischen einem Tier pro 100 Minuten und einem Tier pro 17 Sekunden. Für A. aranciacus ergab sich ein Durchschnitt, der dem bei Cannigione beobachteten gleicht. Wiederum macht sich der Einfluß der Tauchstelle bemerkbar, deutlicher bei $A$. bispinosus als bei $A$. aranciacus. Noch wirksamer ist der Einfluß $\beta$ der Tiefe, auf den die drei Arțen aber verschieden reagieren. 
Die absolute Dichtc wurde an der Costa Colostrai geschätzt, indem auf einem Beobachtungsfeld von $6400 \mathrm{~m}^{2}$ Fläche, das auf 7 bis 8 Meter Tiefe angelegt war, wiederholt alle sichtbaren Seesterne gesammelt wurden. Auf dem Feld fanden sich 37 Individuen von $A$. aranciacus und 105 von $A$. bispinosus. Somit entfällt bei $A$. aranciacus ein Seestern auf $173 \mathrm{~m}^{2}$, bei $A$. bispinosus einer auf $61 \mathrm{~m}^{2}$.

Am gleichen Ort wurden auf 2 bis $5 \mathrm{~m}$ Tiefe mit der Egge $900 \mathrm{~m}^{2}$ durchgekämmt. Bei A. bispinosus belief sich der Ertrag auf 20 Seesterne, bei $A$. jonstoni auf 26 . Somit entfiel bei $A$. bispinosus ein Seestern auf $45 \mathrm{~m}^{2}$, bei $A$. jonstoni einer auf $36 \mathrm{~m}^{2}$.

\section{Häufigkeitsverteilung nach Tiefe}

Von $A$. aranciacus ist bekannt, daß er bis in eine Tiefe von $100 \mathrm{~m}$ vorkommt (KoeHler, 1921; Massé, 1966) und von A. bispinosus, daß er sich in Tiefen zwischen 2 und $50 \mathrm{~m}$ aufhält (KoEHLER, 1921; MAssé, 1966). Unsere Erfahrungen bestätigen diese Angaben und zeigen außerdem, daß da, wo zwei oder mehr Arten an der gleichen Küstenstelle vorkommen, ihre Abundanzmaxima auf verschiedener Tiefe liegen.

Tabelle 3

Relative Seesterndichten auf den Profilen 1 und 2 (P 1, P 2 in Abb. 1b) an der Costa Colostrai in den Jahren 1974 und 1975

\begin{tabular}{|c|c|c|c|c|c|c|c|}
\hline \multirow{3}{*}{$\begin{array}{l}\text { Tiefe } \\
\text { (m) }\end{array}$} & \multicolumn{3}{|c|}{ A. aranciacus } & \multicolumn{3}{|c|}{ A. bispinosus } & \multirow{3}{*}{$\begin{array}{c}\text { A. jonston } \\
1975 \\
\text { P } 2\end{array}$} \\
\hline & & & 1975 & & & 1975 & \\
\hline & $P 1$ & P 2 & P 2 & P 1 & P 2 & P 2 & \\
\hline 1 & & & & & & & 0,01 \\
\hline 2 & & & & & & 0,5 & 3,4 \\
\hline 3 & & & & & & 1,4 & 2,8 \\
\hline 4 & & & & & & 1,0 & 0,2 \\
\hline 5 & 0,4 & 0,2 & 0,2 & 0,6 & 0,7 & 0,8 & 0,2 \\
\hline 8 & 0,5 & 0,3 & 0,3 & 0,4 & 0,6 & 1,4 & \\
\hline 11 & 0,2 & 0,2 & 0,3 & 0,2 & 0,2 & 0,5 & \\
\hline 14 & 0,1 & 0,1 & 0,3 & 0,1 & 0,1 & 0,3 & \\
\hline 17 & 0,3 & 0,3 & 0,5 & 0,1 & 0,2 & 0,1 & \\
\hline 20 & 0,4 & 0,3 & 0,2 & 0,04 & 0,1 & 0,01 & \\
\hline
\end{tabular}

Hier sollen die in Tabelle 3 enthaltenen Ergebnisse nochmals zur Sprache kommen. Abbildung 1b zeigt dic Anlage der zwei Profile, auf denen in den Jahren 1974 und 1975 an der Costa Colostrai Secsterne gezählt und gemessen wurden. Dic Abstände der Sammelstellen vom Ufer waren gegeben durch die Tiefe. Die berücksichtigten Tiefen sind in der ersten Kolonne von Tabelle 3 angegeben. Gesamthaft für alle Wiederholungen und beide Profile sind dic Ergebnisse in Abbildung 2 als Histogramme gezeigt. Bei $A$. jonstoni ist das Vorkommen auf einen schmalen Streifen beschränkt, der von 2 bis $7 \mathrm{~m}$ Tiefe reicht. Die anderen größeren Abundanzen von A. jonstoni, die Tabelle 1 angibt, wurden ebenfalls in untiefen Bereichen festgestellt. Tiefenwärts schließen $A$. aranciacus und $A$. bispinosus an, wobei $A$. bispinosus die größte Abundanz von 3 bis $11 \mathrm{~m}$ Tiefe erreicht und dann seltener wird, während $A$. aranciacus bei $20 \mathrm{~m}$ Tiefe noch nicht selten ist und vermutlich um so dominanter wird, je größer die Tiefe 
ist. Im Bereich von 5 bis $20 \mathrm{~m}$ Tiefe kreuzen sich die Wege beider Arten. Da sich nach den bisherigen Erfahrungen beide Arten von einem ähnlichen Satz an Beutetieren ernähren, sind sie in diesem Bereich Nahrungskonkurrenten. Während $A$. aranciacus in der größeren Tiefe ihr Refugium hat, muß sich $A$. bispinosus im Überschneidungsbereich bewähren. Das System erfährt eine Komplikation, indem A. aranciacus als kräftige, außerdem größte Astropecten-Art A. bispinosus überwältigt und frißt. Es ist anzunehmen, daß sich die ökologischen Nischen der zwei Arten noch in zusätzlichen Dimensionen unterscheiden.

Auf jeder Tiefe ist das Dispersionsmuster nicht zufällig, sondern läßt lockere Aggregate erkennen, die durch dünn besiedelte Flächen voneinander getrennt sind. Die Unterschiede zwischen Profilen bei gleicher Tiefe (Tab. 3) lassen sich als Ausdruck eines solchen Dispersionsmusters auffassen. In der gleichen Bucht nahm die Häufigkeit von $A$. aranciacus nordwärts noch stärker ab (nicht durch Zahlen belegt). Somit hat $A$. bispinosus, zugleich Konkurrent von $A$. aranciacus und sein Beutetier, innerhalb der gemeinsamen Tiefenzone weitere Ausweichmöglichkeiten.

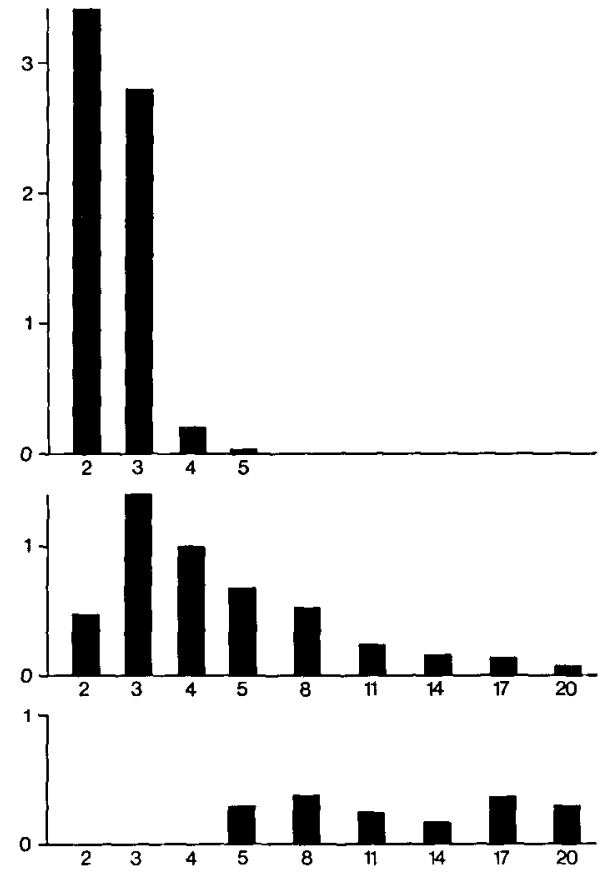

Abb. 2: Verteilung der drei Seesternarten über die Tiefe. Astropecten jonstoni (oben) ist im flachen Wasser häufig, $A$. bispinosus (Mitte) in 4-8 $\mathrm{m}$ Tiefe, noch tiefer $A$. aranciacus (unten). Abszisse: Tiefe in Metern; Ordinate: Anzahl beobachteter Seesterne pro Minute

\section{Körpergrößen von Astropecten aranciacus}

Nach Massé (1966) leben bei $A$. bispinosus die kleineren Seesterne in geringerer Tiefe als die größeren, was bedeuten kann, daß die beiden Größenklassen verschiedene 
ökologische Präferenzen und verschiedene Diäten haben dürften. Eine Folge mag sein, daß die kleineren dem Räubertum der größeren entgehen. Bei verschiedenen Anlässen haben wir jeweils den größten Radius $(R)$ von Seesternen gemessen und die Häufigkeitsverteilung von $R$ für verschiedene Tiefen aufgenommen. Bereits früher (BURLA et al., 1972) konnten wir zeigen, daß sich Seesterne verschiedener Größenklassen ökologisch unterscheiden.

Tabelle 4

Durchschnittlicher Radius $(\overline{\mathrm{R}})$ in $\mathrm{cm}$ von Astropecten aranciacus und A. bispinosus auf dell Profilen 1 und 2 (P 1, P 2 in Abb. 1b) an der Costa Colostrai in den Jahren 1974 und 1975

\begin{tabular}{|c|c|c|c|c|c|c|}
\hline \multirow{3}{*}{$\begin{array}{l}\text { Tiefe } \\
(\mathrm{m})\end{array}$} & \multicolumn{3}{|c|}{ A. aranciacus } & \multicolumn{3}{|c|}{ A. bispinosus } \\
\hline & & & 1975 & & & 1975 \\
\hline & P 1 & P 2 & P 2 & P 1 & P 2 & P 2 \\
\hline 2 & & & & & & 4,1 \\
\hline 3 & & & & & & 4,5 \\
\hline 4 & & & & & & 4,6 \\
\hline 5 & 14,7 & 15,2 & 15,7 & 8,6 & 7,8 & 8,0 \\
\hline 8 & 13,9 & 14,9 & 14,4 & 8,6 & 8,0 & 8,5 \\
\hline 11 & 13,2 & 14,2 & 13,0 & 8,2 & 7,4 & 8,4 \\
\hline 14 & 12,8 & 14,2 & 13,1 & 8,9 & 8,6 & 6,3 \\
\hline 17 & 14,0 & 13,4 & 13,4 & 9,3 & 8,4 & 6,5 \\
\hline 20 & 13,3 & 12,9 & 13,8 & 8,4 & 9,3 & \\
\hline
\end{tabular}

Bei unseren Arbeiten begegneten wir den ganz großen Exemplaren von $A$. aranciacus nur selten und vermuten, ihr üblicher Aufenthaltsort liege in größerer Tiefe. Versuche, bei Cannigione und an der Costa Colostrai eine lineare Abhängigkeit der Körpergröße von der Aufenthaltstiefe nachzuweisen, ergaben allerdings nicht das gewünschte Resultat. Bei Cannigione wurden an sieben Stellen insgesamt 495 Seesterne gemessen und am Fundort außerdem die Tiefe, Sandqualität und Vegetationsverhältnisse protokolliert (Zeilen 5 und 10 in Tab. 1, Tab. 6). In der multiplen linearen Regression, berechnet mit den Programmen BMD02R und BMD03R, kam der Tiefe, die von 4 bis $20 \mathrm{~m}$ variierte, kein gesicherter Einfluß auf die Streuung von $\mathrm{R} z u$. Doch ergab sich, daß die Secsterne auf feinem Sand größer waren als auf Kies; außerdem war die Tauchstelle von Einfluß, was bedeutet, daß die Seestern-Population des Untersuchungsgebiets uneinheitlich war. Eine inverse Proportionalität zwischen der Sandkorngröße und dem Radius (R) kam außerdem in einem Datenmaterial zum Vorschein, das im August 1973 vor Cannigione gesammelt worden war (Burla et al., 1976). Es scheint nun klar zu sein, daß die Körpergröße von Ort zu Ort variiert und die Qualität des Sediments einen bestimmenden Einfluß ausübt. Auf den in Abbildung $1 \mathrm{~b}$ dargestellten Profilen wurden ebenfalls R-Werte gemessen. Die erhaltenen Durchschnitte von $R$ sind in Tabelle 4 enthalten. Die größten Durchschnitte fanden sich in 5 bis $8 \mathrm{~m}$ Tiefe, doch blieben sie bis $20 \mathrm{~m}$ Tiefe nur unwesentlich kleiner. Der Befund bestätigt die bei Cannigione gemachte Erfahrung, daß im strandnahen Bereich die Körpergröße nicht auffällig von der Tiefe abhängt. Lediglich eine Gruppe kleiner Seesterne kommt mit zunehmender Tiefe etwas deutlicher zum Vorschein. Dies geht aus Tabelle 5 hervor, welche die Rohdaten für Profil 1 an der Costa Colostrai in September 1974 wieder- 

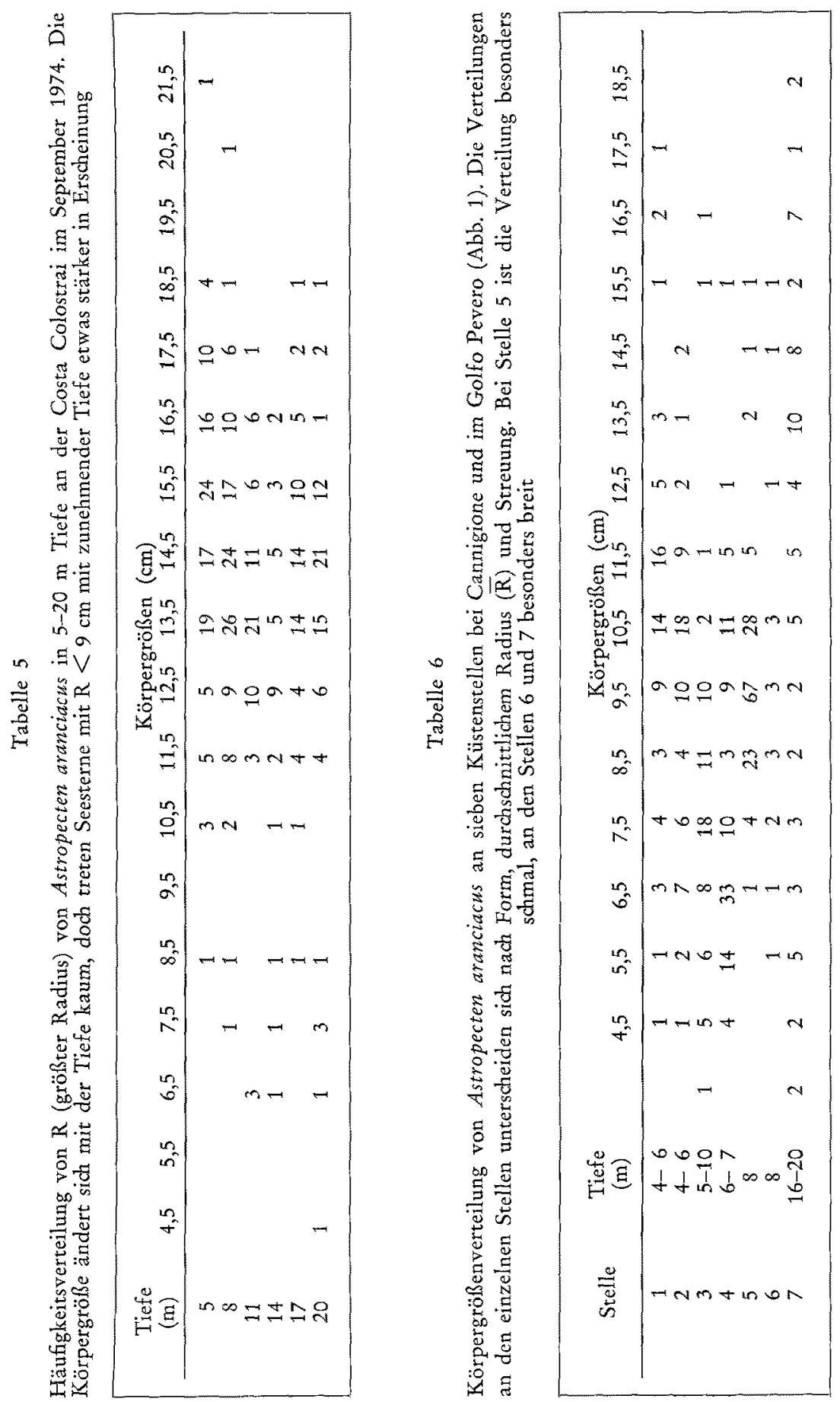
gibt. Rohdaten, die im gleichen Jahr auf Profil 2 und ein Jahr später nochmals auf Profil 1 gesammelt wurden, gleichen denen, die in Tabelle 5 enthalten sind. In Tabelle 6 sind die entsprechenden Rohdaten wiedergegeben, die bei Cannigione im August 1972 gesammelt wurden. Sie zeigen, daß $\mathrm{R}$ an den sieben Sammelstellen ungleich verteilt ist. So erscheint bei Stelle 5 ein besonders schmaler Modus, während bei den Stellen 6 und 7 die Verteilung besonders flach ist. Diese Uneinheitlichkeit scheint typisch zu sein für die Verhältnisse bei Cannigione. Auch unterscheiden sich die Seesterne von Cannigione und Costa Colostrai in der durchschnittlichen Körpergröße. Zwar ist an beiden Orten ein zentraler Modus zu erkennen, der am schlanksten erscheint auf $8 \mathrm{~m}$ Tiefe und seine Lage auf der Abszisse mit der Tiefe kaum ändert. Dieser Modus liegt im Material von der Costa Colostrai bei etwa $15 \mathrm{~cm}$, im Material von Cannigione bei etwa $10 \mathrm{~cm}$. Die beiden Populationen leben unter verschiedenen Bedingungen, und es ist daher nicht zu erwarten, daß die durchschnittlichen Körpergrößen übereinstimmen. Die Flächen bei Cannigione waren relativ kleine, von Posidonienrasen mehr oder weniger umschlossene Sandinseln an nur mäßig exponierten Stellen. Die Korngröße des Substrats zeigte starke lokale Unterschiede, war aber im allgemeinen größer als im Süden. Die Costa Colostrai hingegen ist ein großer, gegen das freie Meer exponierter Strand mit gleichförmig feinem Sand.

\section{Körpergrößen von Astropecten bispinosus}

Beim kleineren $A$. bispinosus sind die Häufigkeitsverteilungen von $\mathrm{R}$ ähnlich breit und flach. Als Beispiel sind die Rohdaten, dic im August 1975 auf Profil 1 gesammelt wurden, in Tabelle 7 vereinigt. Wie bei $A$. aranciacus ist der zentrale Modus auf $8 \mathrm{~m}$ Tiefe am schlanksten und höchsten. Während jedoch bei $A$. aranciacus die Gruppe der kleinen Seesterne mit der Tiefe deutlicher in Erscheinung tritt (Tab. 5), verhält es sich bei A. bispinosus umgekehrt. In Tabelle 7 sind noch Körpergrößen enthalten, die auf

\section{Tabclle 7}

Körpergrößenverteilung von Astropecten bispinosus in 2-14 $\mathrm{m}$ Tiefe auf Profil 1 an der Costa Colostrai (Abb. 1). Im flachen Wasser wurden vorwiegend kleine Seesterne gefunden, tiefer als $5 \mathrm{~m}$ hauptsächlich adulte

\begin{tabular}{|crrrrrrrrrrrrr|}
\hline $\begin{array}{c}\text { Tiefc } \\
(\mathrm{m})\end{array}$ & 1,5 & 2,5 & 3,5 & 4,5 & 5,5 & 6,5 & 7,5 & 8,5 & 9,5 & 10,5 & 11,5 & 12,5 & 13,5 \\
\hline 2 & 1 & 7 & 2 & 3 & 7 & 1 & & & & & & & \\
3 & 3 & 8 & 13 & 14 & 15 & 4 & 2 & & & & & \\
4 & & 15 & 12 & 7 & 17 & 6 & 3 & 2 & & & & \\
5 & & 1 & 2 & 1 & 7 & 2 & 5 & 15 & 10 & 5 & & & \\
8 & & 1 & 6 & 2 & 4 & 6 & 23 & 37 & 52 & 13 & & & \\
11 & & & 4 & 8 & 8 & 6 & 4 & 7 & 12 & 4 & & & 1 \\
14 & & & 9 & 12 & 11 & 6 & 10 & 12 & 7 & 1 & & & \\
\hline
\end{tabular}

Tiefen von 2 bis $4 \mathrm{~m}$ Tiefe gemessen wurden. Offensichtlich handelt es sich um besonders kleine Tiere, eine vom Gros der Adulten tiefenmäßig getrennte Kinderstube. Die von MAssé (1966) erwähnte räumliche Segregation der Größenklassen bei A. bispinosus 
zeigt sich nun auch in unserem Material. Noch deutlicher als bei $A$, aranciacus variiert bei A. bispinosus das durchschnittliche $\mathrm{R}$ innerhalb Tiefe von Profil zu Profil: Auf vier von sechs vergleichbaren Tiefen ist es am größten im südlichsten Profil. Nord- und strandwärts findet man eher kleine Seesterne, süd- und meerwärts eher große.

Meßdaten liegen noch vor von einer Stelle der Costa Colostrai, die $500 \mathrm{~m}$ weiter nördlich als die beiden Profile liegt ( $\mathrm{J}$ in Abb. 1b), sowie von Korsika (B in Abb. 1a). Gesammelt wurde in geringer Tiefe; entsprechend waren die dort angetroffenen Seesterne mehrheitlich klein. An der Costa Colostrai reichte das Vorkommen von 3 bis $8,5 \mathrm{~m}$ Tiefe. Bei breiter Streuung läßt sich doch erkennen, daß strandwärts die kleinen Tiere überwiegen, während die größeren Tiere eher im tiefen Bereich angetroffen wurden.

\section{Körpergrößen und Wachstum von Astropecten jonstoni}

Für die kleinste der drei Arten, A. jonstoni, ist eine bimodale Verteilung typisch, wobei die beiden Modi mehr oder weniger auseinanderliegen. Nur in zwei von sieben Fällen wirkt die Verteilung unimodal. Beim Vergleich der Orte zeigt sich, daß nur im Golfo Pevero Tiere mit $\mathrm{R}=6 \mathrm{~cm}$ auftraten und Tiere mit $\mathrm{R}=5 \mathrm{~cm}$ häufiger waren als an den anderen Orten. An der Costa Colostrai hatte es in $2 \mathrm{~m}$ Tiefe mehr kleine als große Tiere, ein Hinweis darauf, daß sich die Größenklassen lokal ähnlich sondern können wie bei $A$. bispinosus.

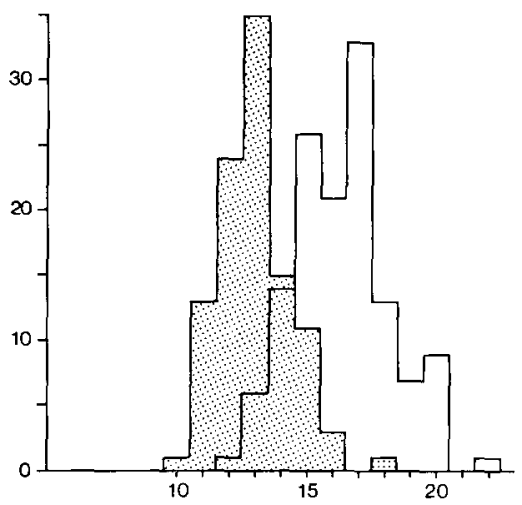

Abb. 3: Größenverteilung der kleinen Individuen von Astropecten jonstoni. Berücksichtigt sind nur Seesterne mit einem Radius kleiner als $25 \mathrm{~mm}$. Punktiert: Muster vom 12. Juni 1975; weiß: Muster an der gleichen Stelle, fünf Wochen später gesammelt. Abszisse: Radius in Millimetern; Ordinate: Anzahl Seesterne

Um Angaben über das Wachstum von A. jonstoni zu erhalten, wiederholten wir an der Costa Colostrai die Messung im Abstand von 5 Wochen. Am 12./13. Juli 1975 sammelten wir in $2 \mathrm{~m}$ Tiefe und setzten die Seesterne am 13. Juli am gleichen Ort wieder aus. Am 19. August 1975 sammelten wir nochmals an derselben Stelle. Abbildung 3 zeigt die Häufigkeitsverteilungen von $\mathrm{R}$, jedoch nur für die Gruppe der kleinen See- 
sterne. Bei der ersten Stichprobe betrug $\overline{\mathrm{R}}$ der kleinen Seesterne $12,04 \mathrm{~mm}$, fünf Wochen später $16,28 \mathrm{~mm}$. Dies entspricht einem durchschnittlichen Wachstum von etwa $4 \mathrm{~mm}$ in 37 Tagen. Bei großen Seesternen veränderte sich der durchschnittliche Radius $\overline{(R)}$ hingegen nicht: Er betrug $36,65 \mathrm{~mm}$ am $12 . / 13$. Juli und $36,67 \mathrm{~mm}$ am 19 . August.

\section{DISKUSSION}

Soweit unsere Erfahrungen über die geographische Verbreitung reichen, bestätigen sie die Angaben früherer Autoren (MAssé, 1966; Koenler, 1921; Tortonese, 1965). Was wir in dieser Beziehung beitragen, sind Fundstellen sowie Angaben über Abundanzverhältnisse und Körpergrößen.

Die relativen und absoluten Bestandesdichten, die wir beobachteten, wirken gering. Sie stimmen jedoch mit den von Massé (1975) gegebenen Werten überein, der für $A$. aranciacus eine Dichte von einem Individuum pro $100 \mathrm{~m}^{2}$ bis zu einem pro $45 \mathrm{~m}^{2}$ feststellte. Auch für andere Seesterngattungen wurden Bestandesdichten geschätzt; in der Größenordnung entsprechen sie unseren Werten. Für Linckia laevigata und Acanthaster planci wurden Dichten von einem Seestern pro $500 \mathrm{~m}^{2}$ bis einem pro $10 \mathrm{~m}^{2}$ angegebenen (LAXTON, 1974), für Acanthaster ellisii eine durchschnittliche Dichte von einem pro $225 \mathrm{~m}^{2}$ (DANA \& WOLFSON, 1970). Dichteangaben für weitere drei Arten bewegen sich $z$ wischen einem Seestern pro $100 \mathrm{~m}^{2}$ und einem pro $10 \mathrm{~m}^{2}$ (BrRKELAND, 1974). Dagegen wurden weit größere Werte in auffälligen Aggregationen verschiedener Echinodermen-Arten beobachtet und Dichten berechnet, die je nach Körpergröße der jeweiligen Art zwischen 50 und 2000 Individuen pro $\mathrm{m}^{2}$ liegen (BRun, 1968, 1969; WARNER, 1969).

Daß Makroinvertebraten des marinen Benthos zoniert vorkommen, ist eine allgemeine Erfahrung (Amouroux, 1972). Dic drei von uns untersuchten Seesternarten zeigen eine Zonierung in bezug auf dic Verteilung der Arten wie auch der Größenklassen. Trotz ausgedehnter Überschncidungsgebicte hat jede Art cine Tiefenzone, in der sie am häufigsten ist. Bei A. bispinosus besteht eine räumliche Sonderung der GröBenklassen nach der Tiefe: Erwachsene Individuen leben in größerer Tiefe als juvenile.

Die Befunde haben eine Bedeutung im Zusammenhang mit Konkurrenz, Kannibalismus, Nahrungsangebot oder einer von Art zu Art verschiedenen Prägung auf abiotische Faktoren. Welche Erklärung zutrifft, lassen die Daten nicht erkennen.

Die Segregation der Größenklassen, wie wir sie bei A. bispinosus beobachteten, wurde auch für Dendraster excentricus beschrieben (MERILl \& Hobson, 1970): Kleine Individuen waren häufig in Strandnähe, die größten fanden sich in ca. $9 \mathrm{~m}$ Tiefe.

Die Beobachtung und Folgerung, daß die kleine Größenklasse von A. jonstoni pro Sommermonat etwa $4 \mathrm{~mm}$ linear wächst, ist ein erster Befund dieser Art für eine Astropecten-Art in natürlichen Verhältnissen. Vergleichbar sind aber Größenverteilungen und Wachstum bei Luidia sarsi (FENCHEL, 1965) und Asterias mbens (SMrTH, 1940). Für beide Arten erstreckte sich die Beobachtungszeit über mehr als ein Jahr. Bei Luidia sarsi wurde die Entwicklung einer Population von der Metamorphose an über zwei Jahre verfolgt. In dieser Zeit erreichten die Seesterne eine Armlänge von 
6 bis $10 \mathrm{~cm}$, was einer mittleren Wachstumsgeschwindigkeit von $4 \mathrm{~mm}$ in 5 Wochen entspricht. Dieser Wert stimmt mit unserem Befund an A. jonstoni gut überein.

\section{ZUSAMMENFASSUNG}

1. An der Ostküste Sardiniens wurden die Abundanzen sowie die Dichte und die GröBenverteilung von drei Seesternarten der Gattung Astropecten mittels Gerätetauchen untersucht. Alle drei Arten leben auf mobilen Böden. Zwei davon, A. bispinosus und $A$. jonstoni, wurden nur auf feinem Sand angetroffen, $A$, aranciacus auch auf Kies. Die Arten sind unterschiedlich zoniert nach Tiefe: die kleinste Art, $A$. jonstoni, lebt im flachen Wasser, während die größte, $A$ aranciacus, am weitesten in die Tiefe vordringt. Die Überschneidungszonen sind breit; an mancher Stelle kommen zwei oder alle drei Arten zusammen vor. Für $A$. aranciacus wurden Dichten zwischen einem Seestern pro $173 \mathrm{~m}^{2}$ und einem pro $25 \mathrm{~m}^{2}$ gemessen, für $A$. $b i$ spinosus von einem Seestern pro $45 \mathrm{~m}^{2}$, für A. jonstoni von einem Seestern pro $36 \mathrm{~m}^{2}$.

2. Die Häufigkeitsverteilungen des Radius ( $\mathrm{R}$ ) von $A$. aranciacus an verschiedenen Stellen unterscheiden sich nach Form, Mittel und Streuung. Im Bereich von 4 bis $20 \mathrm{~m}$ scheint sich die Körpergröße mit der Tiefe nur unsystematisch zu verändern. Hingegen waren die Individuen auf feinem Sand im Durchschnitt größer als auf Kies.

3. Bei A. bispinosus stellten wir eine Segregation der Größenklassen nach der Tiefe fest: die kleinen Seesterne waren im flachen Wasser, die großen tiefer unten zu finden. Die Körpergrößenverteilung von A. jonstoni war an den meisten Orten bimodal. Im Sommer nimmt der Radius der kleinen Individuen in fünf Wochen um etwa $4 \mathrm{~mm} z u$.

Danksagungen. Die Aufenthalte am Meer wurden ermöglicht durch Beiträge der CarlHescheler-Stiftung und der Georges- und Antoine-Claraz-Schenkung. Während der Abfassung des Manuskripts war einer der Autoren (G. RIBI) Empfänger eines vom Schweizerischen Nationalfonds zur Förderung der wissenschaftlichen Forschung geleisteten Salärs. Mehrere Tauchkollegen, unter ihnen H. MAAG, Taucher am Zoologischen Museum der Universität Zürich, halfen beim Sammeln der Daten. Frau C. Hesse-Honegger zeichnete die Abbildungen.

\section{ZITIERTE LITERATUR}

Amouroux, J.-M., 1972. Données sur la structure et l'instabilité des peuplements infralittoraux de la côte du Roussillon. Thèse, Université de Paris, 102 pp.

Birkeland, C., 1974. Interaction between a sea pen and seven of its predators. Ecol. Monogr. $44,211-232$.

Brun, E., 1968. Extreme population density of the starfish Asterias rubens L. on a bed of Icelands scallop, Chlamys islandica (O. F. MülLER). Astarte 32, 1-4.

- 1969. Aggregation of Ophiothrix fragilis (ABIldgaArd) (Echinodermata: Ophiuroidea). Nytt. Mag. Zool. 17, 153 160.

- Ferlin, V., Pabst, B. \& Ribr, G., 1972. Notes on the ecology of Astropecten aranciacus. Mar. Biol, 14, 235-241. 
Burla, H., Pabst, B. \& Stahel, W., 1976. Vorkommen von Astropecten aranciacus (Asteroidea, Echinodermata) in Abhängigkeit von Umweltbedingungen. Helgoländer wiss. Meeresunters. 28, 167-182.

Christensen, A. M., 1970. Feeding biology of the sea-star Astropecten irregularis Pennant. Ophelia 8, 1-134.

Dana, T. F. \& Wolfson, A., 1970. Eastern Pacific crown-of-thorns starfish populations in the lower Gulf of California. Trans. San Diego Soc. nat. Hist. 16, 83-90.

- NEWMAN W. A. \& FAGER, E. W., 1972. Acanthaster aggregations: interpreted as preliminary responses to natural phenomena. Pacif. Sci. 26, 355-372.

Fenchel, T., 1965. Feeding biology of the sea-star Luidia sarsi Düben \& Koren. Ophelia 2, 223-236.

Gulliksen, B. \& Skjaeveland, S. H., 1973. The sea-star, Asterias rubens L., as predator on the ascidian, Ciona intestinalis L., in Borgenfjorden. North-Troendelag, Norway. Sarsia 52, 15-20.

KOEHLER, R., 1921. Echinodermes. Faune Fr. 1, 1-210.

Laxton, J. H., 1974a. A preliminary study of the biology and ecology of the blue starfish Linckia laevigata (L.) on the Australian Great Barrier Reef and an interpretation of its role in the coral reef ecosystem. Biol. J. Linn. Soc. 6, 47-64.

- 1974b. Aspects of the coral-eating starfish Acanthaster planci. Biol. J. Linn. Soc. 6, 19-45.

MassÉ, H., 1975. Ethologie alimentaire de Astropecten aranciacus L. In: 9th European marine biology symposium. Ed. by H. Barnes. Aberdeen Univ. Press, Aberdeen, 343-355.

- 1966. Contribution à l'écologie du genre Astropecten Linck. Recl. Trav. Stn. mar. Endoume 41, 187-191.

Merill, R. J. \& Hobson, E. S., 1970. Field observations of Dendraster excentricus, a sand dollar of Western North America. Am. Midl. Nat. 83, 595-624.

PAINE, R. T., 1969. The Pisaster-Tegula interaction: prey patches, predator food preference, and intertidal community structure. Ecology 50, 950-961.

Smith, G. F. M., 1940. Factors limiting distribution and size in the starfish. J. Fish. Res. Bd Can. 5, 84-103.

Tortonese, E., 1965. Fauna d'Italia. Echinodermata. Calderini, Bologna, 422 pp.

Warner, G. F., 1969. Brittle-star beds in Torbay, Devon. Underwater Ass. Rep. 4, 81-85.

Anschrift des etstgenannten Autors: G. Rubr

Zoologisches Museum

Künstlergasse 16

$\mathrm{CH}-8006$ Zürich

Schweiz 